\title{
Deficiency of Adenosine Deaminase 2 (DADA2): One Disease, Several Faces
}

\author{
Pallavi Pimpale Chavan ${ }^{1}$ (D) $\cdot$ Divya Ramadoss $^{1}$ (D) Archana Khan $^{1}$ (D) $\cdot$ Pui Y. Lee $^{2}$ (D) Raju Khubchandani $^{1}$ (D)
}

Received: 29 January 2021 / Accepted: 10 May 2021 / Published online: 31 May 2021

(C) Dr. K C Chaudhuri Foundation 2021

To the Editor: Mutations in the adenosine deaminase 2 (ADA2) gene cause autosomal recessive monogenic vasculitis syndrome [1]. Besides our first report from India [2], there is scanty data on pediatric cases from Asia [3, 4]. We now have a series of 10 cases diagnosed over the last 2 y (Table 1 ).

With a 7:3 male to female ratio and a wide age range of presentation, the table also shows the varied settings in which the pediatrician should suspect the condition. Fever with polymorphic rash (livedoid, maculopapular, petechial, pruritic, ulcerative, subcutaneous nodules or erythema nodosum rash -7 patients), chronic abdominal pain (6), diarrhea (3), and a granulomatous adenitis treated as tuberculosis could well be presentations to a primary practitioner. Referrals from and involvement of neurologists (recurrent stroke - 3, mononeuritis multiplex - 2), hematologists (pure red cell aplasia - 1, unexplained anemia - 2, or cytopenias - 2), orthopedic surgeons (bone dysplasia mimic - 1), ophthalmologists (sudden onset blindness - 1) and even general or specialist surgeons (intestinal perforation - 2, gastric bleed -
1, perinephric hematoma - 1), or a nephrologist (hypertension - 3) testify to the wide phenotypic spectrum of DADA2 and the need to sensitize various specialists.

ADA2 enzyme assay performed in 4 patients showed it to be deficient in all. The p.G47R mutation was the commonest in our series and was seen in all 4 patients who hailed from the endogamous Agarwal community. The disease is very responsive to etanercept with $9 / 10$ patients being adherent, steroidfree, and in complete remission with normal activities of daily living. Treatment with tumor necrosis factor blockade (etanercept or adalimumab) has been safe and progressively affordable with etanercept biosimilar. Residual hypertension may be seen with renal involvement and 1 patient who contracted COVID-19 and recovered uneventfully.

With endogamy and consanguinity prevalent in India, we recommend pediatricians/subspecialists to keep a searching eye for this potentially fatal but very treatment-responsive systemic autoinflammatory disease, with many disguises.

Pallavi Pimpale Chavan

drpallavipimpale@gmail.com

1 Section of Pediatric Rheumatology, NH SRCC Children's Hospital, 1-1A, Keshavrao Khadye Marg, Haji Ali, Haji Ali Government Colony, Mahalakshmi, Mumbai 400034, Maharashtra, India

2 Division of Allergy, Immunology and Rheumatology, Boston Children's Hospital, Boston, Massachusetts, USA 


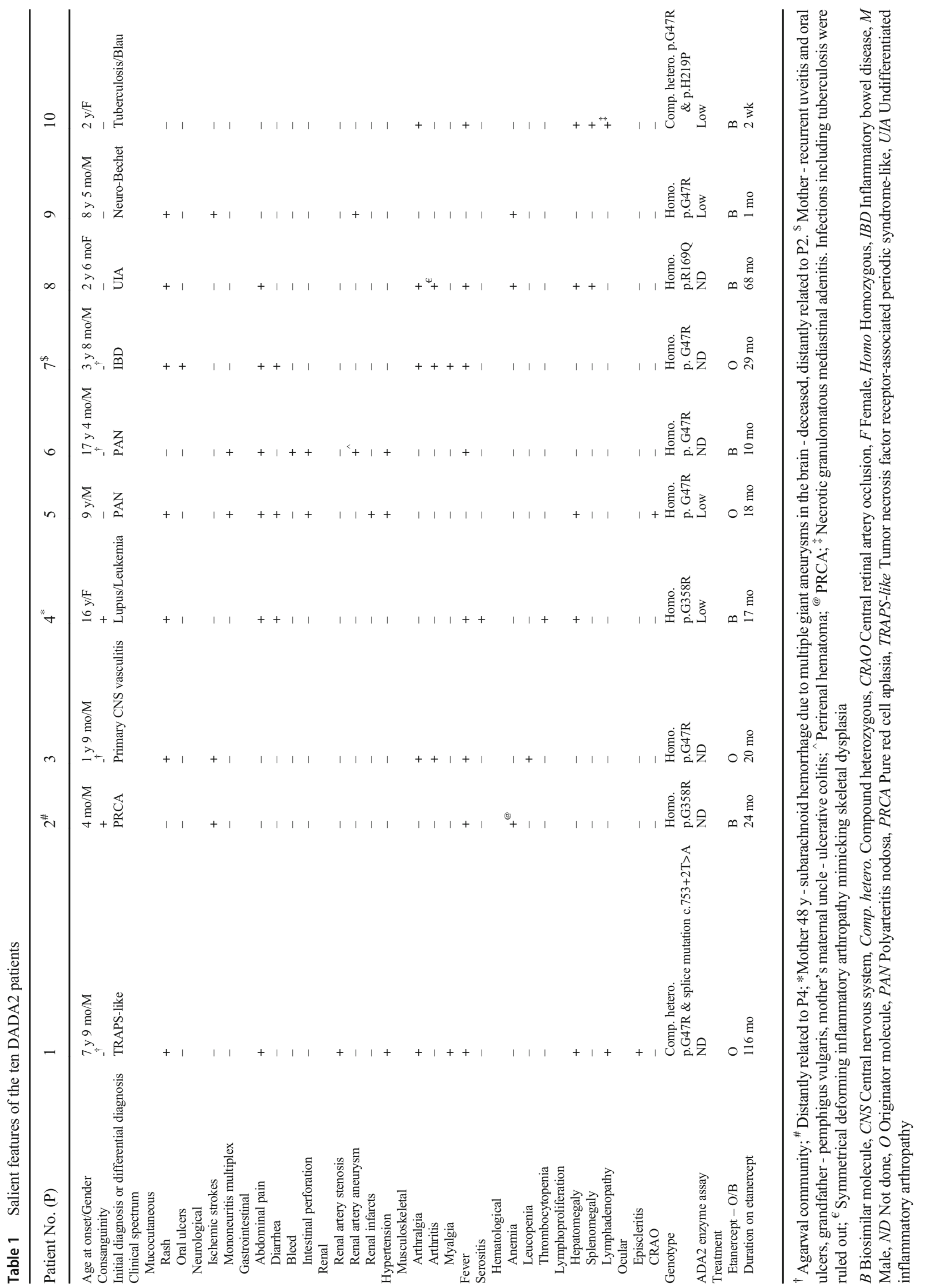




\section{Declarations}

\section{Conflict of Interest None.}

\section{References}

1. Zhu Q, Yang D, Ombrello AK, et al. Early-onset stroke and vasculopathy associated with mutations in ADA2. N Engl J Med. 2014;370:911-20.
2. Khubchandani R, Aksentijevich I. Deficiency of adenosine deaminase 2 (DADA2) - a new autoinflammatory disease with multisystem features. Indian Pediatr. 2020;57:1074-5.

3. Sharma A, Naidu G, Sharma VV, et al. Deficiency of adenosine deaminase 2 (DADA2) in adults and children: experience from India. Arthritis Rheumatol. 2021;73:276-85.

4. Suri D, Rawat A, Jindal A, et al. Spectrum of systemic autoinflammatory diseases in India: a multi-centric experience. Front Immunol. 2021;12:630691.

Publisher's Note Springer Nature remains neutral with regard to jurisdictional claims in published maps and institutional affiliations. 